\title{
Flexibility and Resiliency of Electric Power Systems: Analysis of Definitions and Content
}

\author{
Nikolai Voropai ${ }^{1}$ and Christian Rehtanz ${ }^{2}$ \\ ${ }^{1}$ Melentiev Energy Systems Institute, 130 Lermontov Str., 664033 Irkutsk, Russia, voropai@isem.irk.ru \\ 2Dortmund University of Technology, 70 Emil-Figge Str., D-44227 Dortmund, Germany, christian.rehtanz@tu- \\ dortmud.de
}

\begin{abstract}
This paper deals with actively studied terms "flexibility" and "resiliency" of electric power systems (EPS). Definitions and content of these new fundamental EPS properties are analysed. Objective trends in development of EPS with regard to these properties are discussed. Possible measures for improving flexibility and resiliency of EPS are suggested.
\end{abstract}

Key words: Electric power systems, flexibility, resiliency, survivability, definitions, measures.

\section{Introduction}

Electric power systems (EPSs) are constantly developing complex entities using innovative electricity production, transmission, distribution and storage technologies, which are affected by a variety of objective factors. The process of development changes the properties of these systems and causes the need to update new properties of the transformed electric power systems. This is associated with the new problems arising in the operation and expansion of these systems and, accordingly, with the need to use new means to ensure normal operation and effective expansion of EPSs. This paper deals with the analysis of two new terms "flexibility" and "resiliency" of EPS, and the reasons why it is necessary to consider these new properties and to develop possible measures for improving flexibility and resiliency of EPS.

The paper is constructed as follows. In Chapter 2, we will discuss different definitions of EPS flexibility and suggest a general definition. Chapter 3 includes the explanation of possible measures for improving EPS flexibility. Objective trends in developing EPSs, which reduce their flexibility level, are discussed in Chapter 4. The understanding of resiliency is presented in Chapter 5. Chapter 6 deals with the discussion of preferable measures for improving EPS resilience. The conclusions include the main results of this paper and future works.

\section{Flexibility definition}

In the past few years, authors of many works have been actively discussing a new term reflecting the transformation of EPS properties. This is flexibility [1 -
4, etc.]. Let us note several different definitions of EPS flexibility for understanding this problem.

Flexibility of operation - the ability of a power system to respond to the changes in demand and supply is a characteristic of any EPS with high levels of gridconnected variable renewable energy (primarily, wind and solar) [1].

Operational flexibility is the capability of the EPS to absorb disturbances for maintaining a secure operating state. Locational flexibility is the operational flexibility at the given bus in the grid [2].

Supply and demand uncertainties of EPSs increase dramatically because of more variable resources are integrated into them. This requires the system to have the ability to react to a sudden change and accommodate new state within acceptable time period and cost [3].

A technical system is flexible when its control system can guarantee that all requirements and constraints are met at each time moment of operation under changes in internal and external factor [4].

As we see, the understanding of flexibility as an important property of EPS is more or less similar. Generally speaking, taking into account the basic sense of the above mentioned and the other definitions, it is possible to consider the following definition and content of EPS flexibility.

The flexibility of the electric power system is its ability to maintain normal state when exposed to internal (sudden changes and fluctuations of load, power flows through the lines, and generation) and external (sudden disturbances) random (undetermined, uncertain) factors.

Sudden changes in load are caused by its random irregular fluctuations due to the variability in electricity demand of many consumers, uncertainty of the spot (balancing) electricity market and activity of consumers managing their electrical consumption online. Power 
flows along the lines can change online unpredictably under the influence of random irregular load fluctuations and changes in electricity prices and contracts for electricity sale/purchase in the spot (balancing) electricity market. Random changes in generated power are a characteristic of renewable energy sources, primarily, wind turbines, as well as small hydropower and photovoltaic power plants. Sudden disturbances include the operation of protection and emergency devices, false actions of personnel, etc.

It is interesting to compare the flexibility property with the traditional term "security" of EPS. Considering [16], security of EPS refers to the degree of risk in its ability to survive imminent disturbances (contingencies) without interruption of customer service. It relates to robustness of the EPS to imminent disturbances and, hence, it depends on the electric power system operating condition as well as the contingent probability of disturbances.

Security of EPS is a rather complicate property from the point of view of the studied problems and aspects of interests [17]. There are different types of security deterministic and probabilistic, static and dynamic. Different considerations can be used to form the sets of studied scenarios of operating conditions, disturbances, control actions, etc.

For the customers, an acceptable level of EPS security means the guaranteed electricity supply. Therefore, it is possible to define it as security of supply to customers. In this sense, EPS flexibility provides the security of electricity supply.

\section{Measures to provide the EPS flexibility}

To provide the flexibility of electric power system, we can consider the capabilities of increasing the flexibility of generation, electrical network and load, and using the possibilities of protection and control system. Let us discuss these problems in more detail.

- Frequency characteristics of speed regulators and inertia of rotating machines play an important role for the level of flexibility of traditional generation as the measures of self-adaptation to uncertain factors [5]. Generation flexibility can be provided by increasing the speed of units loading and unloading processes, and their range; maintaining the necessary level of operational generation reserve and increasing fuel supply reliability for power plants [1 - 3].

- Flexibility of transmission and distribution electrical networks can be increased by eliminating weak points in the network and increasing the efficiency of using the transfer capabilities of the lines, in particular, by applying smart grid technologies, and providing the required level of transfer capability margins. There are new measures to increase flexibility of active electrical networks by dynamic and adaptive topology reconfiguration [6, etc.].

- Flexibility of load can be increased by using voltage and frequency regulation effects as the measure of self-adaptation to uncertain factors [5], by involving demand-side management and using local electricity storages, distributed generation facilities at consumers, and other local devices $[1,2,7$, etc.]. New possibilities of increasing load flexibility can be fulfilled by prosumers [8].

- Integrated multi-energy systems give new additional measures to increase EPS flexibility by using heat storages, heat pumps and electrical boilers $[9,10$, etc.]. Innovative gas systems in integrated electric power and gas systems are a good possibility for meeting electric power systems flexibility requirements by using more effective distributed generation units $[10,11]$.

- Efficient protection and control systems play a key role in increasing EPS flexibility [12, 13], including protection and control of EPS in multi-energy systems [14]. The efficiency of systems to control EPS operating conditions can be enhanced by using innovative smart technologies, improving the forecast accuracy of state variables, reducing the time of generation of control actions and increasing their frequency, etc. [1 - 3].

It is necessary to note a very detailed survey paper [15] (393 references). The authors discuss the following measures to increase the EPS flexibility: demand-side management; grid ancillary services (different reserves, black-start, etc.); energy storages; supply-side flexibility (power plant response, combined-cycle gas turbines, etc.); advanced technologies (electricity-to-thermal, vehicle-to-grid, etc.); grid infrastructure; electricity markets.

It is important to note the necessity of using market mechanisms to effectively implement the EPS flexibility measures [18]. Let us discuss this aspect for power plants. From the perspective of an individual asset owner, power plant operational flexibility could be important for minimizing short- and long-run costs and for maximizing revenue. From a system perspective, flexibility is useful for increasing reliability, lowering system costs, and integrating renewables.

\section{Trends in the EPS flexibility}

Modern electric power systems are characterized by a sufficient level of flexibility due to their internal properties of self-adaptation, self-stabilization, and control of operating conditions. Self-adaptation of an EPS, i.e. its ability to withstand internal and external destabilizing factors, is achieved due to the existence of load-based voltage and frequency regulation effects and frequency characteristics of generation. Another factor ensuring the self-adaptation of EPS is its inertia determined by the inertia of rotating mechanical mass of rotors of power generators. Owing to these effects, EPS to a certain extent adapts to sudden changes in its state and external impacts, meanwhile the control systems cope with these changes and impacts when the state variables of the system go beyond certain limits, by bringing these variables within the specified boundaries of the feasibility region of EPS operation [5].

The electric power systems of the 21 st century are expected to face dramatic changes in their internal structure and properties that will significantly reduce their self-adaptation and self-stabilization, and hence the 
flexibility of these systems. Internal factors are associated with the massive use of power electronics and rectifier-inverter systems for connecting high-frequency small gas turbines, wind generators, photovoltaic systems, energy storage devices, DC lines and links, frequency-controlled motors, local rectifiers of many electrical loads to electric power systems. This significantly reduces the above-mentioned load-based voltage and frequency regulation effect and generationbased frequency regulation ability, as well as the EPS inertia. On the other hand, the planned rapid growth in the number of intermittent generators, especially wind turbines, will lead to a significant increase in the negative impact of output fluctuations on the power system capabilities to self-adaptation, and, accordingly, reduce their flexibility [5].

At the same time, the control systems of many devices with power electronics (FACTS, energy storage systems, DC lines and links, etc.) are highly efficient and their wide use will significantly increase the controllability of future EPSs.

Thus, there is a tendency towards reduction in the flexibility of future EPSs, an increase in the level of instability of states of these systems, and at the same time, towards an increase in their controllability. The combination of individual factors and their levels vary depending on EPS structure and properties. However, the above-mentioned general trends are the same. The prevalence of negative factors and underestimation of positive ones in the process of EPS development and operation will lead to an unacceptable reduction in the flexibility of these systems, an increase in their failure rate, a decrease in the reliability of electricity supply to consumers and deterioration in power quality. Thorough comprehensive studies are necessary to deal with the negative factors and effectively use the positive factors.

\section{Resiliency definition}

Recently, a new term, resiliency, reflecting EPS properties transformation has been actively discussed and studied [19 - 24, etc.]. The most comprehensive definition of resiliency is given in [20], where it is defined as "the ability of the system to withstand a change or a disruptive event by reducing the initial negative impacts (absorptive capability), by adapting itself to them (adaptive capability) and by recovering from them (restorative capability)". This definition refers to any systems irrespective of their nature. In [22], the discussed property is addressed as a complex one, including ecological, organizational and system components. The latter features as an ability to minimize the value and duration of deviations from target metrics of a system.

The studies presented in $[19,21,23,24]$ address the resiliency with reference to EPSs, and in [21, 23], the focus is on extreme external events (for example, hurricanes, etc.). The problem of cyberattacks is also studied as potential external disturbances affecting the information-communication subsystem of present-day cyber-physical EPSs [25 - 27, etc.]. At the same time, in $[19,21,24$, etc. $]$, the authors consider it as the problem of EPS resiliency in relation to the cascading system blackouts. Let us draw attention to the increase in scale of after-effects of the cascading system failures for customers in time based on the statistics about the USA EPSs over 1991 - 2005 (see Figure 1) [19]. This tendency of growth of scale of after- effects of system blackouts is true for any developing EPS. Figure 2 shows the main system behaviors in different situations from a resiliency point of view [28].

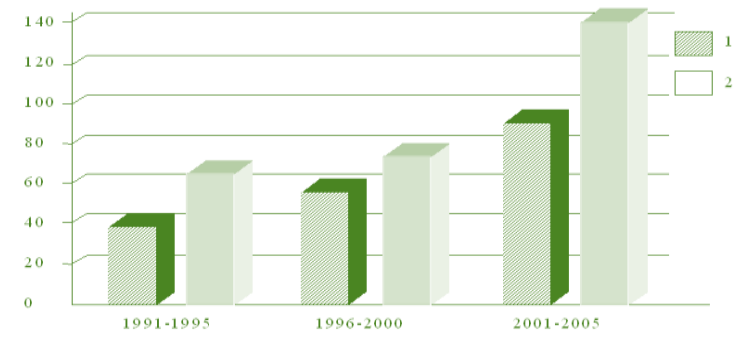

Fig.1. Tendency in EPS blackouts problem: 1 - The number of outages affecting more than 50000 consumers; 2 - The number of outages greater than $100 \mathrm{MW}$

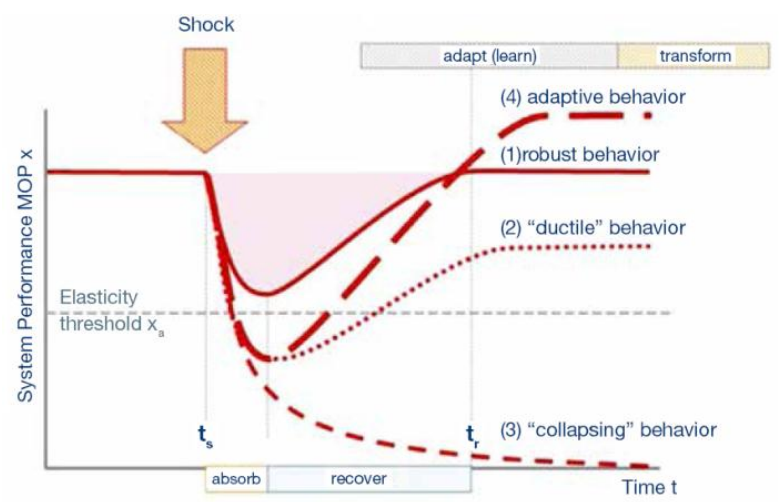

Fig. 2. Main regularities of the system stable and unstable behaviors.

The term corresponding to resiliency is survivability. Applicable to EPS, survivability is a property of a system to withstand disturbances without allowing their cascade development with a mass interruption of electricity supply to consumers, and to recover the system initial state or one close to it [29,30]. From this definition, one can see, that the survivability property includes the absorptive and adaptive abilities of a system noted in [20] as well as its ability to recover.

In some literature (e.g., [31]) the term "vulnerability" is used instead of "survivability". Vulnerability is related to a dynamic mode of reliability, to dynamic security. The differences between the terms "survivability" and "vulnerability" are the following: survivability involves a certain "activity" of a system resisting perturbations due to rationally organized structure, expedient operating conditions, and efficient control and protection. Vulnerability reflects a certain "passive" response of a system to perturbations. In general sense, vulnerability is a complementary ("opposite") property of a system as compared to survivability $[29,30]$. 
As of correlation flexibility and resiliency, the flexibility provides the absorptive and adaptive capabilities of EPS.

It is expedient to note some details related to EPS survivability concept using Figure 3 . There are two

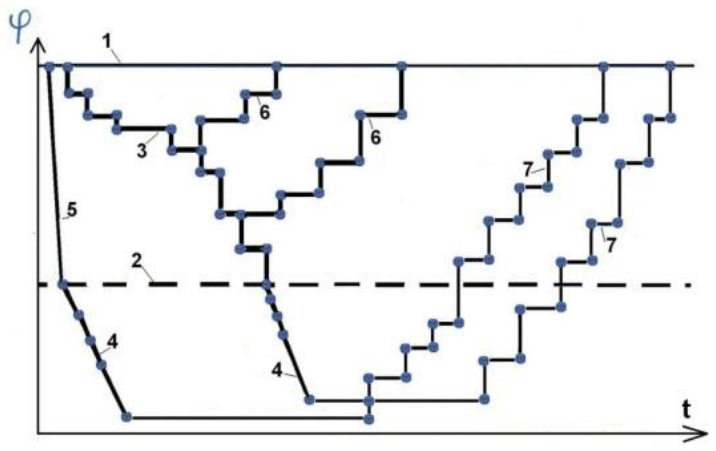

Fig. 3. Illustration for a EPS behavior in terms of survivability: 1 - normal state, 2 - limiting state, 3 cascading process, 4 - catastrophic cascading, 5 - extreme disturbance, 6 - preventing from cascading process, 7 restoration stage

possible cases. In the first case, an emergency process starts with the ordinary perturbation, and then, due to protection failures and personnel mistakes, there occurs a cascading development of emergency (see 3 in Figure 3). In each stage of such development, the control and protection systems attempt to interrupt the cascading development (see 6 in Figure 3). Upon reaching the limiting state 2 , the emergency cascade becomes irreversible (see 4), cascading development is rapid, the emergency protection and control schemes either have no time to response, or have already exhausted their possibilities by this stage. Then, restoration stage follows the post-emergency state.

The other case is related to an extreme non-ordinary initial disturbance (see 5 in Figure 3), after which the system appears below the limiting state, and the uncontrollable development of an emergency occurs.

It is interesting to note the relations between flexibility and resiliency of EPS. Taking into account the above- mentioned definitions and sense of these terms, flexibility of EPS provides its resiliency creating absorptive capability.

There are some similar aspects in the properties of resiliency and survivability of EPS. We can compare elasticity threshold and limiting state, collapsing behavior and catastrophic cascading, adaptive behavior and restoration, robust behavior and preventing from cascading in the definitions of resiliency and survivability, respectively.

\section{Measures to provide EPS resiliency / survivability}

Generally speaking, the following measures can be taken to provide EPS resiliency / survivability:

- Develop reliability and security standards for considering them during expansion planning and operation of EPS. It is important to note the necessity of specific reliability standards for the problems of resiliency / survivability in the case of extreme disasters considering power supply requirements for essential consumers.

- Build effective wide-area emergency protection and control systems. These systems are very important to improve first of all the resiliency/survivability of EPS in the case of cascading emergencies.

- Establish effective restoration procedures. There are some differences in implementation of of these procedures for EPS restoration after cascading process and after extreme disaster. In the first case, the matter is in recovering only EPS operating conditions, in the second case, it is necessary to additionally change failed equipment.

- Organize regular dispatcher training. This measure is important for both cases, but the content of training is different for each of them.

- Generalize of the cascading events. This work is very important first of all for such emergencies. It is necessary to note that each EPS system emergency with cascading development is unique but the knowledge of some general mechanisms gives the possibility of decreasing the probability and negative consequences of such emergencies.

Principally, there are two different ideologies to prevent cascading development of EPS emergencies in terms of the main role of dispatcher or automatic protection and control systems. International practice shows that automatic interruption of cascading process is preferable because of a rapid character of such a process and stress conditions for dispatcher in the majority of incidents and high probability of false actions of dispatcher under such conditions [13, 30, 32, etc.].

\section{Economic aspects of flexibility and resiliency/survivability of EPS}

The problems of EPS flexibility and resiliency/survivability are both technical and economic. However, the economic sides of these problems are different, as well as the technical specifics.

Considering flexibility as an economic problem, it is necessary to take into account the following clear positions:

a) Flexibility increasing measures require additional investment and operating costs.

b) There are specific objectives to minimize the above-mentioned costs during EPS expansion planning and operation.

c) Economic mechanisms of flexibility measures implementation can be based on the ancillary services markets.

To determine the economic sense of resiliency/survivability is more difficult. It is clear, that it is necessary to have investment and operating costs to provide absorptive, adaptive and restorative capabilities of the EPS. As for cascading system emergencies, their probabilities are very low but their consequences for consumers are highly negative. Therefore, it seems that the main measures have to provide the cascading process 
interruption based on effective protection and control systems, and the fast restoration of the EPS according to reliability and security standards. Considering the resiliency to extreme disasters, additional design measures for EPS expansion are necessary taking into account specific standards requirements. Fast restoration of EPS in this case is also important.

\section{Conclusion}

There are opposite trends in the EPS development towards a decrease in their flexibility and resiliency and towards an increase in their controllability. Comprehensive studies are necessary to consider the negative factors and effectively use the positive factors to provide acceptable operation of future electric power systems.

\section{References}

1. Cochran J., Miller M., Zinaman O., Milligan M., e.a., "Flexibility in $21^{\text {st }}$ Century power systems", $21^{\text {st }}$ Century Power Partnership, Denver, USA, Clean Energy Ministerial, 2014, pp. 1-14.

2. Bucher M.A., Delikaraoglou S., Heussen K., Pinson P., Andersson G., "On quantification of flexibility in power systems", 2015 IEEE Power Tech, Eindhoven, Netherlands, June 29 - July 2, 2015, 6 p.

3. Jinye Zhao, Tongxin Zheng, Litvinov E., "A unified framework for defining and measuring flexibility in power system", IEEE Trans. on Power Systems, 2016, Vol. 31, No. 1, pp. 339-347.

4. Volin Yu.M., Ostrovskiy G.M., "Flexibility analysis of complex technical systems under uncertainty", Avtomatika I Telemehanika (Remote Control), 2002, No. 7, pp. $92-106$.

5. Voropai N.I., Osak A.B., "Electric power systems of the future", Energeticheskaya Politika, 2014, Issue 5, pp. $22-29$ (in Russian).

6. Sun Hongbo, Wang Yishen, Nikovski D., Zhang Jinyun, "Flex-Grid: A dynamic and adaptive configurable power distribution system", 2015 IEEE Power Tech, Eindhoven, Netherlands, June 29 - July 2, 2015, $6 \mathrm{p}$.

7. Schuitema G., Ryan L., Aravena C., "The consumer's role in flexible energy systems", IEEE Power and Energy Magazine, 2017, Vol. 15, No. 1, pp. $53-60$.

8. Casey E., Beaini S., Pabi S., Zammit K., Amarnath A., "The triple bottom line for efficiency", IEEE Power and Energy Magazine, 2017, Vol. 15, No. 1, pp. $34-42$.

9. Kiviluoma J., Heinen S., Qazi H., Madsen H., Strbac G., etc., "Harnessing flexibility from hot and cold", IEEE Power and Energy Magazine, 2017, Vol. 15, No. 1 , pp. $25-33$.

10. Chen Xinyu, Kang Chongquing, O’Maley M., Xia Qing, etc., "Increasing the flexibility of combined heat and power for wind power integration in China: Modeling and implication", IEEE Trans. on Power Systems, 2015, Vol. 30, No. 4, pp. 1848 - 1857.
11. Heinen S., Hewicker C., Jenkins N., McCalley J., O'Malley M., etc., "Unleashing the flexibility of gas”, IEEE Power and Energy Magazine, 2017, Vol. 15 , No. 1 , pp. $16-24$.

12. Gopalan S., Sreeram V., Iu H., Mishra Y., "A flexible protection scheme for an islanded multi-microgrid", $4^{\text {th }}$ IEEE PES Innovative Smart Grid Technologies Europe, Copenhagen, October 6-9, 5 p.

13. Voropai N.I., Efimov D.N., Kolosok I.N., Kurbatsky V.G., Glasunova A.M., Korkina E.S., Tomin N.V., Panasetsky D.A., "Intelligent control and protection in the Russian electric power system", Application of Smart Grid Technologies, London, UK, ElsevierAcademic Press, 2018, pp. 61 - 140.

14. Dall'Anese E., Mancarella P., Monti A., 'Unlocking flexibility", IEEE Power and Energy Magazine, 2017, Vol. 15, No. 1, pp. $43-52$.

15. Lund P.D., Lindgren J., Mikkola J., Salpakari J., "Review of energy system flexibility measures to enable high levels of variable renewable electricity", Renewable and Sustainable Energy Reviews, 2015, Vol. 45, pp. 785 - 807, Ref, 393.

16. Kundur P., Paserba J., Viter S., "Overview on definition and classification of power system stability", CIGRE / IEEE PES Int. Symposium on Quality and Security of Electric Power Delivery Systems, 2003, 8 p.

17. Marceau R.J., Endrenyi J., Allan R., Alvarado F.L., e. a., "Power system security assessment: A position paper", Electra, 1997, No. 175, pp. $49-77$.

18. Bistline J.E., "Turn down for what? The economic value of operational flexibility in electricity markets", IEEE Trans. Power Systems, 2019, Vol. 34, No. 1, pp. $527-534$.

19. Amin M., "Challenges in reliability, security, efficiency, and resilience of energy infrastructure: Toward smart self-healing electric power grid", IEEE PES General Meeting, Pittsburg, USA, July 20 - 24, 2008, 5 p.

20. Cen Nan, Sansavini G., Kroeger W., "Building an integrated metric for quantifying the resilience of interdependent infrastructure systems", $9^{\text {th }}$ Int. Conference on Critical Information Infrastructure Security, Limassol, Cyprus, October 13 - 15, 2014, $12 \mathrm{p}$.

21. Wang Y., Chen C., Wang J., Baldick R., "Research on resilience of power systems under natural disasters - A review", IEEE Trans. Power Systems, 2016, Vol. 31, No. 2, pp. $1604-1612$.

22. Wang Z., Nistor M.S., Pickl S.W., "Analysis of definitions of resilience", $20^{\text {th }}$ IFAC World Congress, Toulouse, France, July 9 - 14, 2017, pp. 11136 11144.

23. Panteli M., Mancarella P., Trakas D.N., Kyriakides E., Hadziargiriou N.D., "Metrics and quantification of operational and infrastructure resilience in power systems", IEEE Trans Power Systems, 2017, Vol. 32, No 6, pp. $4732-4741$.

24. Kezunovic M., Overbye T.J., "Off the beaten path: Resiliency and associated risk", IEEE Power and Energy Magazine, 2018, Vol. 16, No. 2, pp. 26 - 35 . 
25. Mehrdad S., Mousavian S., Madraki G., Dvorkin Yu., "Cyber-physical resilience of electrical power systems against malicious attacks: A review", Current Sustainable / Renewable Energy Reports, https://doi.org/10.1007/s40518-018-0094-8

26. Voropai N., Kolosok I., Korkina E., "Resilience assessment of the state estimation software under cyberattacks", E3S Web of Conferences, 2018, Vol. 58, https://doi.org/10.1051/e3conf/2018580.2013

27. Papkov B.V., Kulikov A.L., Osokin V.L., "Cyber threats and cyberk in electric power industry", Nizhny Novgorod, 2017, 80 p. (in Russian).

28. Heinimann H.R., "Future resilient systems", Singapore-Zurich, ETH Risk Center, 2014, 600 p.

29. Voropai N.I., "The problem of large electric power system survivability", IEEE Power Tech, Stockholm, Sweden, June 18 - 22, 1995, 5 p.
30. Besanger Y., Eremia M., Voropai N., "Major grid blackouts: Analysis, classification, and prevention", Handbook of Electrical Power System Dynamics: Modeling, Stability, and Control; Ed. By M.Eremia and M.Shahidehpour, Hoboken, IEEE Press-Wiley, 2013, pp. $789-863$.

31. Fouad A.A., Zhou Qin, Vittal V., "System vulnerability as a concept to access power system dynamic security", IEEE Trans. Power Systems, 1994, Vol. 9, No. 2, pp. 1009 - 1015.

32. Sovalov S.A., Semenov V.A., "Emergency protection and control in power systems", Moscow, Energoatomizdat, 1988, 416 p. (in Russian).

This work was supported by Russian Scientific Foundation, project \# 19-49-04108, and by the German Research Council (DFG), project \# RE 2930/24 